\title{
An Elderly Person has Come to You - How Can he Help him?
}

\author{
Viktoriia Yu Zharinova* \\ The Institute of Gerontology, Ukraine \\ *Corresponding author: Viktoriia Yu Zharinova, The Institute of Gerontology, Kyiv, Ukraine

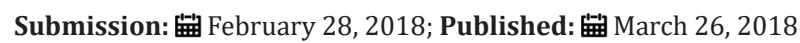

\section{Editorial}

One of the most difficult problems of geriatric cardiology is the problem of adherence of elderly patients to the treatment process usually long and complex.

The concept of "adherence" includes the clarity of the patient's compliance with recommendations for medication and the patient's interaction with the doctor, including a response to his psychological support. That is, adherence is an active voluntary joint with the physician participation of the patient in the process of optimizing his treatment in order to obtain a better therapeutic result.

From the point of view of adherence, patients may be rejecting treatment (not wishing to be treated) treated periodically (in this case, the "percent coincidence of goals" between the doctor and the patient is considered) and constantly adhering to medical recommendations. Of course, the best adherence to therapy is achieved if the patient correctly assesses the nature of his illness and the risks associated with it and trusts the treating doctor.

This is most important in the case of geriatric patient treatment, since, as a rule, an elderly person refers to life quite pessimistically is not able to adequately assess the real state of his health and does not have sufficient motivation for treatment. This means that the primary responsibility for the patient's subsequent attitude to his condition and the prescribed therapy lies primarily on the physician and to treat the elderly patient, the doctor must with all the responsibility of the person holding someone else's life in his hands.

It follows from the foregoing that the most important method of increasing adherence to therapy in geriatric practice is a constructive dialogue between the doctor and the patient aimed at increasing motivation for treatment, fighting fears, discussing the consequences of active treatment and abandoning it. The doctor should help the patient to choose the right position, strengthen his confidence in the need for medication and the possibility of recovery. A very important role in the formation of a positive attitude is also played by the patient's family - their moral, and often material support, can play a decisive role in the future fate of the elderly person. Therefore, it is highly desirable that in a conversation between a doctor and a patient, close people participate, whose opinion is especially important for the patient.

The next no less important factor in the formation of a high adherence to therapy is the correct choice of the regimen for taking medications of their quantity and dose. It should be remembered that an elderly person usually has three or four chronic diseases requiring simultaneous treatment. A typical, average statistical example of such a patient can be a patient with IHD and hypertension having dyscirculatory encephalopathy, concomitant diabetes mellitus, chronic gastritis, cholecystitis and pancreatitis suffering from constipation. Therefore, after visiting several doctors, the patient receives a list of recommended medications, consisting of 8-12 medicines to take each of which should be 1-2 times a day, focusing on the dependence on food intake and time of day.

This polypharmacy is characteristic for the treatment of the elderly all over the world. For example, in Germany, $40 \%$ of elderly patients take 4 or more medicines. In the US, the average patient aged over 65 years receives an average of 10.7 new or renewable prescriptions. In the US and the UK, elderly patients account for one third of the total number of drug users. In Italy, $40 \%$ of people over 70 years old take from 4 to 6 drugs daily, and another $12 \%$ - more than 9 drugs, that is, more than half of the Italian elderly are daily victims of polypharmacy.

The need to take a large number of medications and a complex scheme of therapy lead to a significant reduction in adherence to treatment in this category of patients. Firstly, this is due to the peculiarities of the physiology of the elderly, including: a decrease in the total volume of fluid in the body, including the volume of circulating blood, leads to an increase in the concentration of drug substance in the body. Reduced levels of albumin in the blood, causing an increase in the concentration of free (active) drug in the blood. Reduction of the volume velocity of blood flow in organs, including kidneys, which reduces renal filtration. Decrease in the efficiency of enzymatic systems of the liver, which causes an increase in the concentration in the blood of drugs that undergo biotransformation into inactive metabolites with the help of these systems. Reduction of intensity of motility of the gastrointestinal tract, leading to increased absorption of drugs. Decreased body 
weight due to muscle and fat tissue, which reduces the volume of distribution of medicines; a decrease in the specific number of receptors (including in the central nervous system).

In these conditions, the patient's responsible attitude to medical appointments is likely to lead to the development of a number of side effects and complications. It is shown that the incidence of complications of drug therapy reaches $5 \%$ in the older age group. The consequences of these complications are 3-55 of all hospitalizations of the elderly. Therefore, when giving advice to an elderly person, in addition to detailed information about the drugs, their dose and the regimen, the doctor must warn about possible side effects of treatment, the presence and nature of inter-drug interactions.

This factor is very important for further adherence to treatment and maintaining the authority of the doctor, since even in the case of a good tolerability of medicines, an elderly person must have a detailed knowledge of the instructions to them and, in the case of even minimal complications, may question the doctor's competence and his sincere interest in his fate.

However, another development of events is possible: the patient will exhibit psychosocial features of behavior-frightened by the number of appointments or become entangled in them, will not believe in doctors, and without motivation to continue treatment, interest in life and excess funds will not be treated at all. Obviously, no matter how events develop, the result of a "standard" approach to the treatment of an elderly patient is, as a rule, refusal to continue treatment, which leads to the progression of diseases, an increase in the number of complications

After all, it is proved that in elderly people, low adherence to therapy is a predictor of an unfavorable outcome of diseases. Thus, in the population of patients older than 65 with diabetes, hyphenension and dyslipidemia, which included 137 thousand of patients, the incidence of heart failure, associated hospitalization, and the cost of treatment were significantly lower in regularly treated patients than in untreated patients.

In the Heart and Soul study, it was shown that the incidence of vascular events in patients adherent to treatment was half that of non-drug users. According to the results of this study, adherence to therapy was recognized as an independent predictor of acute vascular events in this category of patients.

Deterioration of the clinical course of diseases naturally increases the costs of recovery and significantly increases the cost of treatment for older people

Therefore, to increase the adherence of the elderly to prescribed therapy, it is necessary to reduce the number of prescribed drugs to the maximum effective minimum. A vivid illustration of this situation was the results of monitoring the therapy of patients in 76 European clinics who showed that $80 \%$ of patients took it once, if the dose of the drug required 3-4 times, adherence to admission was reduced to $51 \%$. Another independent factor of compliance for the elderly is the number of tablets taken per day. The analysis of the therapy of more than 11.9 million patients showed that the use of fixed combinations of drugs contributed to an improvement in adherence to medical therapy by $26 \%$, and thus was a factor in improving the life expectancy of patients. From this point of view, the modern concept, aimed at creating combined drugs, is likely to be the most realizable among elderly patients. Although, on the other hand, a significant drawback of this approach for this category of patients may be the risk of side effects due to the need to take fixed doses of the drugs included in the combination, or individual "insufficiency" or "redundancy" of individual components.

Thus, in a study in India comparing the clinical efficacy of a fixed combination of low-dose hydrochlorothiazide, acetylsalicylic acid, atenolol, ramipril, and simvastatin, it was shown that the effect of HR and blood pressure on the combination drug did not differ from the mono preparations. At the same time, its effect on the lipid spectrum and aggregation activity of platelets was not sufficient . Therefore, the basis of medical art in the treatment of an elderly patient should not be an attempt to create a "universal pill". And the use of a minimum amount of medicines with maximum feasibility and effectiveness.

Another important mechanism to increase adherence of older people to drug therapy is regular monitoring of compliance with medical recommendations. Given that most elderly people suffer from forgetfulness, it is necessary that all instructions for the elderly person be issued in writing, written briefly and clearly. The features of therapy and its scheme should be discussed at every contact with the patient, as often as possible. It is advisable to control the number of medications taken and the regimen for each visit at each visit, since, according to international studies, forgetfulness is the main factor in the violation of adherence to therapy in the elderly.

That is why the strategy of "short-term recommendations" or "advice" having a short period of urgency is more effective for older people, which means that it is better to take control and allow more frequent monitoring of the regimen for taking prescribed medications. A very important role for the patient in therapy is played by the position of the middle and junior medical personnel of the clinics. Well-trained and properly oriented staff can significantly improve patient adherence to therapy, not only in the hospital, for example through telephone contacts.

In a two-year, randomized controlled trial involving 400 uncompleted patients who received five or more drugs for chronic illness, regular contact with clinic staff (not by doctors) resulted in a $41 \%$ reduction in patient mortality over a 3 -year follow-up period, which significantly correlated with the improvement of adherence to therapy against the background of weekly telephone communication. The study showed that weekly contact with clinic staff led to better results than an increase in the cost of treatment.

Drug-dispensed medicine is an effective way to increase adherence to treatment in the elderly. This method is widely and effectively distributed in Europe and consists in creating individual 
multi-dose tablets for medications structured by day of the week and time of day. Having such an individual tablet, the patient, his relatives or medical personnel can easily understand what medicines at what time the patient should take, whether he took the next dose and when he should take the next one. Of course, the use of such tablets greatly facilitates the process of treatment for an elderly person, improves its quality.

This method was tested on 200 elderly patients with arterial hypertension and dyslipidemia, taking on average 9 tablets per day. Prior to the start of the observation, the patients' adherence to therapy was $61 \%$. After acquaintance with the use of individual tablets in 6 months of follow-up, adherence to therapy increased to $97 \%$. Increased adherence to therapy was associated with normalization of blood pressure and blood lipid levels.

After the end of the study, in patients who stopped using plates, adherence to therapy decreased to $65 \%$, those who continued to use them remained at 95\%. In a similar controlled trial involving more than 2,500 people over the age of 65 , it was shown that arranging an orderly approach to taking medication is a more effective way of improving the quality of treatment for older people than social and psychological programs.

In support of this, in Denmark, an analysis of the case histories of 19,000 patients receiving "safe blister" therapy (individual tablets) showed that in 6 months of follow-up, the hospitalization rate of patients older than 80 years decreased from 7 to $4 \%$ and the duration of their hospitalizations decreased from 7- 9 to 5-6. For 6 months of follow-up, the number of days of hospitalization in this category of patients decreased by $62.5 \%$ compared to the same period before the introduction of this drug-use chain.

Thus, maintaining adherence to treatment in elderly patients is a very difficult task, for a more successful solution which the doctor should remember that:

A. Notall diseases need drug therapy; there are other ways of correcting health disorders (diet, psychotherapy, lifestyle changes, quitting smoking and other bad habits).

B. If possible, avoid symptomatic therapy, focusing on etiotropic, and if this is not possible, then on pathogenetic therapy.

C. Before prescribing drug therapy it is necessary to evaluate compatibility, potentiation of side effects, total amount of drugs.

D. To strive for the appointment of a minimum number of drugs with supposedly maximum effectiveness,

E. If possible, simplify the scheme of taking medications.

F. Give the patient clear written recommendations for taking drugs and as often as possible to monitor their performance.

G. Involve the middle and junior medical staff of the clinics in the treatment of the elderly person, and in the outpatient settings of the patient's relatives.

H. Remember that the primary responsibility for the elderly person's commitment to therapy and, ultimately, the quality and duration of his life depends on the qualifications and human qualities of the doctor.
Creative Commons Attribution 4.0 International License

For possible submissions Click Here

\section{Submit Article}

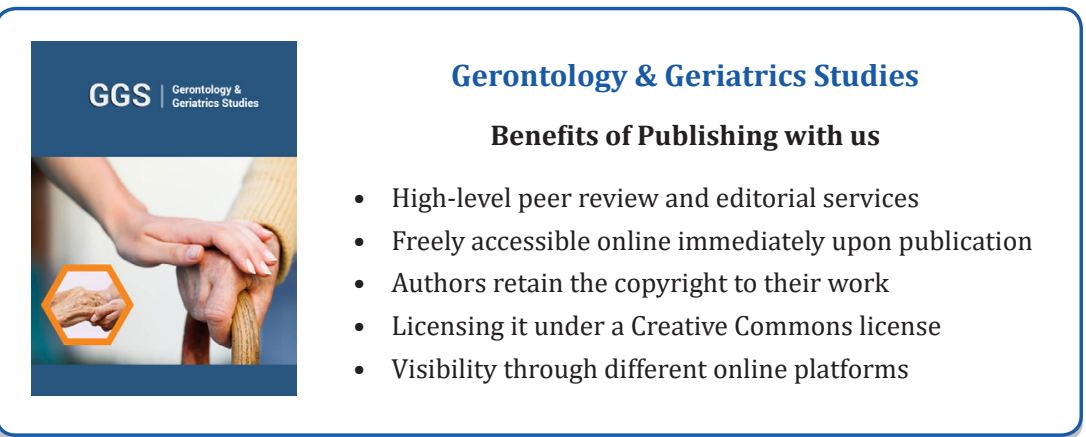

